\title{
La intimidación entre los escolares y nuevas formas de violencia
}

Norman D. Pautasso ${ }^{1}$

\section{Artículo}

Material original autorizado para la publicación en la revista Psicodebate. Facultad de Ciencias Sociales. Universidad de Palermo.

Recibido 17-03-2015 | Aceptado 14-10-2015

\section{Resumen}

El presente artículo recopila algunos resultados de varios estudios que abordaron la problemática de la Intimidación y/o Acoso entre los niños/as de edad escolar en instituciones de educación general básica de la región central de la Provincia de Santa Cruz- Argentina. Expresa ciertas consideraciones teóricas respecto de la cuestión de la intimidación entre los niños/as y sobre la violencia en las escuelas. Ofrece datos de la región presentando información sobre los comportamientos agresivos más comunes, las formas que según los propios niños originan sus conductas violentas y los lugares en los se producen tales actos. Describe las poblaciones escolares que padecen este tipo de prácticas y analiza algunas diferencias respecto de los ciclos escolares, sexo y características zonales. Se extrae además el índice de victimización y el índice de agresión general de las diversas muestras obtenidas en la región. A modo de discusión, el trabajo aporta cierta descripción acerca de algunos fenómenos producidos por el acoso en la región, como el caso del "efecto de acorralamiento", generado por la falta de anonimato en lugares de baja población. Presenta nuevas problemáticas emergentes en el campo de investigación sobre el tema como el surgimiento de nuevas formas de violencia, refiriendo a las prácticas que se producen mediante recursos como la mensajería instantánea, el correo electrónico, los chat, las blogs, fotologs, redes sociales, todos los relacionados con tecnologías de comunicación digital. Para culminar ofrece algunas ideas acerca de la construcción de Dispositivos de Alerta Temprana y Dispositivos de Intervención Temprana.

Palabras Clave: bullying, intimidación, acoso escolar, maltrato infantil, violencia, cyber bullying.

1 Universidad Nacional de la Patagonia Austral; UNPA - Argentina; normanpautasso@gmail.com 


\section{Abstract}

This article is intended to collect some results of the several studies that have been made concerning Bullying and Harassment among boys and girls who attend basic education institutions in the central part of Santa Cruz province, in Argentina. It encloses theoretical framework about the problem of bullying and violence among children at school. It presents information about the region, some common aggressive behaviors as well as different ways and places in which those violent habits might be produced, according to children. Describes school populations suffering from such practices and analyzes some differences from school years, gender and zonal characteristics. It also shows samples of Victimization Rate and General Aggression Rate got in the area. Open to further discussion, this work provides certain description about some bullying phenomenon in the area, such as the case of the "Round-up Effect", produced by the lack of anonymity in barely populated places. This article presents new emerging issues in the research field, like the appearance of new ways of violence, related to digital communication technologies such as instant messaging, e-mail, chat, blog, photoblog and social networks. To conclude, it offers some ideas about the construction of Early Warning Devices and Early Intervention Devices.

Keywords: : bullying, harassment, violence, child maltreatment, cyber bullying. 
Este trabajo es producto de los resultados de varios estudios que abordan la problemática de la intimidación entre los niños en edad escolar en escuelas de educación general básica de la región central de la Provincia de Santa Cruz-Argentina.

La intimidación entre los niños en edad escolar es una problemática que en los últimos tiempos vislumbra una creciente preocupación de muchos expertos en la violencia que algunos niños ejercen sobre otros; y según plantea Giberti (2000): "la incapacidad de los mayores para detectar estos casos propicia el desborde violento del amenazado". La cuestión de la intimidación puede considerarse uno de los problemas relacionados con la violencia escolar, más específicamente con la violencia en la escuela.

El fenómeno de la intimidación es conocido en el ámbito europeo y norteamericano como bullying, que podría traducirse al castellano como intimidación o acoso. Olweus (1998), definió sencillamente la situación de intimidación o acoso planteando que: "un niño es agredido o se convierte en víctima cuando está expuesto, de forma repetida y durante un tiempo, a acciones negativas que lleva a cabo otro alumno o varios de ellos" (p. 25), entendiendo en este contexto que una acción negativa involucra una intención de herir, causar daño o incomodar a otro niño. A los fines del presente trabajo se tomarán como sinónimos los conceptos de intimidación, acoso y Bullying.

Giberti (2000) establece una distinción entre intimidación y amenaza. La intimidación se caracteriza por su persistencia en el tiempo. Por lo general se habla de niños que amenazan a otros niños, transformando la intimidación en amenaza. Sin embargo, se trata de hechos diferentes. El término intimidación deriva del verbo temer y es una acción con fines de acobardar a otro, reducirlo a la pasividad produciéndole temor; se asocia a la conducta de los matones. Existe diferencia entre la amenaza y la intimidación, pero la primera puede convertirse en la segunda cuando se sistematiza y se convierte en recurrente. "Se trata de una relación víctima-victimario que excede la definición de conflicto: enfocarlo como tal arriesga equivocar el diagnóstico". Es un comportamiento prolongado de rechazo social, hostigamiento y/o agresividad física entre los propios alumnos que se convierten en víctimas de sus compañeros. Se trata de relaciones de dominio-sumisión en las que determinados alumnos emplean prácticas abusivas para someter y controlar a otros. Tanto Voors (2006) como Sabucedo y Sanmartin (2007) y otros especialistas mencionan que, para considerarlas como intimidación, bullying o acoso, es necesario que cumplan ciertos criterios básicos como ser: la existencia de un patrón constante de hostigamiento (reiteración), un agresor en clara ventaja, una víctima en clara desventaja o en una situación de victimización. Suele incluirse también un público que presencie lo ocurrido (espectadores activos o pasivos). 
Como describen Harris y Petrie (2006), la intimidación se compone de comportamientos directos, por ejemplo, molestar, atormentar, amenazar, golpear o asaltar, que son realizados por uno o más estudiantes en contra de una víctima. Además de los ataques directos, la intimidación puede ser más sutil, como, por ejemplo, causar el aislamiento social de un alumno a través de la exclusión social intencional. Mientras que los niños típicamente se involucran en los métodos más directos de intimidación, las niñas que provocan este tipo de agresión comúnmente utilizan métodos más indirectos y sutiles como, por ejemplo, propagar rumores injuriosos e imponerle el aislamiento social a su víctima. Estas mismas conclusiones son avaladas por numerosos estudios realizados en países diversos (Hopkins, Taylor, Bowen \& Wood, 2013; Pörhölä \& Kinney, 2010). Sea directa o indirecta la intimidación, su componente principal es que la agresión física o psicológica ocurre repetidas veces, creando así un patrón constante de hostigamiento y abuso.

El maltrato entre iguales incluye variados comportamientos de acoso, burlas y ridiculizaciones, amenazas, insultos, robo de propiedades, agresiones físicas y verbales de diferente gravedad, hablar mal de los demás, vandalismo, chantaje, prácticas de exclusión o marginación ignorando a la víctima e impidiendo su participación social. En general, muchos de estos comportamientos no suelen ser identificados como una forma corriente de violencia por los docentes. Las autoras Garay y Gezmet (2001) afirman que existen con mucha fuerza en nuestras escuelas y que suelen sufrir una suerte de "naturalización" como una forma corriente de trato.

Bejarano y Recio (2000) establecen la siguiente distinción con respecto a otros fenómenos que es preciso diferenciar de las prácticas de intimidación: "es necesario valorar y distinguir el problema de la victimización entre iguales de las malas relaciones entre escolares, aunque ambos cursen con algunas características semejantes. Las malas relaciones son un problema más generalizado pero menos intenso". Los autores mencionados entienden que hay temas relacionados con la indisciplina o el mal comportamiento que afectan la convivencia escolar pero consideran que tales no son problemas serios de violencia.

Allá por el año 2000, el fenómeno del acoso en la escuela había tomado gran preocupación en el contexto internacional, especialmente en el ámbito europeo en países como Holanda, España, Francia, Suecia, Irlanda, Bélgica, y otro largo listado de naciones. En el ámbito americano había una importante producción particularmente en EEUU, pero recién alrededor del 2003 comienzan a emerger estudios en países latinoamericanos. En Argentina, en el año 2004, se crea el "Observatorio Argentino de Violencia en las Escuelas", con el fin de estudiar la problemática de la violencia en contextos escolares. Desde el Observatorio Argentino de Violencia en la Escuela así como del Observatorio de Convivencia 
Escolar (este último conformado en el año 2006) se han realizado aportes muy valiosos en lo que se refiere al análisis de la violencia en el país. Sus producciones marcan una dirección en el tema que instaló en la agenda argentina un compromiso de abordar con seriedad las situaciones de violencia en las escuelas. Publicaciones de autores como Miguez (2009) dan cuenta de ello. Los aportes de los observatorios fueron relevantes para la elaboración y aprobación de "Ley para la promoción de la convivencia y el abordaje de la conflictividad social en las instituciones educativas - Ley 26.892", promulgada en octubre de 2013. Estos avances no están exentos de debate acerca del fenómeno del acoso escolar o bullying, ya que el mismo equipo del Observatorio produjo un documento donde planteó su posición sobre el tema proponiendo una necesaria reformulación del concepto (Lejarraga, Pampurro, Zajac, \& D'odorico, 2011). La preocupación del Observatorio se orientó hacia el mejoramiento de la convivencia escolar. Respecto de los datos estadísticos que recopiló hay que reconocer que ha logrado conformar a la fecha una base informativa muy importante de diversas provincias del país nunca antes lograda. Aunque tales referencias están disponibles en varios documentos (Catala, 2008; Noel, 2009), no hay detalles precisos sobre las zonas del interior de las provincias en las que se recaudó información y no es posible visualizar si en las localidades más pequeñas y de baja población se obtuvieron datos.

En la última década los estudios sobre la problemática han pasado de evaluar la incidencia y grado de la intimidación a estudiar las consecuencias emocionales de este trato injusto. Entre las consecuencias devastadoras para el sujeto infantil se han demostrado por ejemplo: baja autoestima, una imagen negativa de sí mismos y relaciones inseguras con otras personas. También se han establecido vínculos entre pensamientos suicidas, depresión e intimidación y algunos niños han manifestado pensar en auto lesionarse o en ejercer violencia contra los demás (Bauman, Tomey, \& Walker, 2013; Cerezo Ramírez, 1998; Cerezo Ramírez, 2001; Kowalski \& Limber, 2013).

Se comparte la idea de Sabucedo y Sanmartin (2007) de que las prácticas intimidatorias son difíciles de detectar y las víctimas de las mismas aún más. Suele ser más frecuente identificar a los victimarios que a las víctimas, lo que pone a los maestros y padres en la instancia final del proceso cuando la situación ya puede estar produciendo efectos nocivos. Los profesores y aún los padres suelen no atender a las situaciones que presentan estas prácticas, suelen restarle importancia o desvalorizar sus posibles efectos.

En resumen, las prácticas de intimidación entre los niños son una realidad presente en los escenarios escolares, además de su constancia en otros contextos de la vida infantil. Esta realidad frecuentemente enfrenta a los psicólogos con casos de niños que se niegan a concurrir a la escuela, niños atemorizados, con 
pánico de salir de la escuela o de la casa por temor a algún tipo de agresión. En otros casos, con niños que se atreven a desafiar la amenaza y terminan siendo víctimas o victimarios de reacciones de violencia física mucho más graves.

Contribuir a dimensionar la problemática de la intimidación en la escuela, a identificarla, a estudiarla, a prevenirla, es apuntar a que los niños aprendan en un mejor ambiente escolar, despojado de amenazas y acosos que los angustien, los atemoricen, les provoquen ansiedad o dañen su salud. Aun cuando los resultados proporcionaran datos de que es un fenómeno restringido en la región, la tarea sería satisfactoria si colaborara en la prevención de la problemática y el abordaje de los afectados. Se estará apuntando hacia una educación que garantice el respeto y la aceptación entre compañeros, es decir, una formación basada en la tolerancia, sustento de cualquier sociedad que tenga como horizonte sostener la vida democrática.

Este escrito toma como fuente tres estudios de campo realizados en la región central de la Pcia. de Santa Cruz, Argentina. Las preguntas generales que originaron tales estudios fueron: ¿Qué dimensión tienen en la región las conductas de acoso, intimidación o bullying entre los escolares? ¿Cuáles son los índices de victimización y de agresión general? ¿Qué tipos de conductas de intimidación predominan? ¿Cuáles son los motivos y dónde se originan las agresiones entre los niños que asisten a la escuela?

El objetivo general fue explorar y describir las prácticas de intimidación en niños de la Escuela General Básica en la región central de la provincia de Santa Cruz, Argentina. Los objetivos específicos consistieron en: (a) evaluar el nivel de intimidación en los escolares mediante la obtención de índices de victimización e índices de agresión general; (b) analizar diferencias entre los índices de agresión general e índices de victimización en las ciudades de la región; (c) evaluar diferencias en los índices según la edad de los niños; (d) analizar si existe una asociación entre el sexo y los tipos de agresiones; (e) indagar si el sexo se relaciona con algunas de las conductas que originan más agresiones entre los escolares; (f) proponer dispositivos y estrategias que permitan combatir el problema del acoso escolar y producir conocimiento sobre el tema.

\section{Método}

\section{Participantes}

Los estudios combinaron una metodología cualitativa e instrumentos cuantitativos. El primer estudio de campo se realizó en escuelas situadas en las localidades de San Julián, Piedrabuena y Puerto Santa Cruz, durante los años 2005 y 2006. Se utilizó un muestreo no probabilístico, consistente en la selección de 2 cursos por año, desde tercer año del primer ciclo a noveno del tercer ciclo de la EGB en 
cada institución. Participaron completando el cuestionario un total de 868 alumnos cuyas edades fueron entre 8 y 17 años, siendo el $48 \%$ mujeres y $52 \%$ varones, y 6 de los participantes no proporcionaron datos sobre sexo. Del total de la población escolar de los grados 3ro. a 9no. la muestra significó para el caso de las escuelas de San Julián una cifra cercana al $40 \%$, para el caso de Piedrabuena un $60 \%$ y para Pto. Santa Cruz un $80 \%$ del total de alumnos. El segundo estudio de campo en la región se realizó durante el año 2009 en la totalidad de los grados del 3er. Ciclo de una escuela en una localidad de la región, obteniendo una muestra no probabilística de 264 estudiantes entre los 13 y 17 años de edad. La muestra representó el $85 \%$ de total de los matriculados en los mencionados cursos, el 53\% fueron varones, el $47 \%$ mujeres. El tercer estudio de campo en el año 2010 fue de tipo cualitativo y efectuado en las localidades de Pto. Santa Cruz, Piedrabuena y Gobernador Gregores. Se obtuvo una muestra no probabilística de 564 niños entre 6 y 16 años de edad, un $47 \%$ de varones y $53 \%$ de mujeres, todos asistentes a las escuelas de EGB y Polimodal de poblaciones de la región central de la Pcia. de Santa Cruz.

\section{Materiales}

Tanto en el primer estudio de campo de los años 2005/2006 como en el segundo en 2009, se recopila información para estimar el grado y tipo de intimidación en niños de escuelas de educación general básica. Para ello se utilizó un cuestionario readecuado de Sharp y Smith (1994), que los autores titularon "Mi vida en la Escuela”. La readecuación se realizó por cuestiones de lenguaje en algunos de sus ítems (se modificaron varios términos propios del uso en España que se consideraron inapropiados para la cultura argentina), respetando el sentido original del indicador. Tal instrumento es ampliamente utilizado en el campo investigativo para medir la violencia escolar. Aun cuando posee relativa antigüedad, muchos estudiosos la consideran una herramienta válida para el análisis de las conductas de acoso entre iguales. Su constructo contiene los indicadores necesarios para la evaluación del bullying y de otras prácticas de violencia entre los niños en la escuela. El instrumento definitivo presentó 38 ítems, del original sólo se eliminó el ítem "durante esta semana otro chico/a me dejó algo", por decisión del equipo de investigadores que consideró que el ítem presentaba aspectos muy confusos. Se mantuvieron 13 indicadores de la dimensión de "clima positivo" y el total de los 25 ítems que miden el "clima negativo". Presenta un formato de escala Likert en el que los niños deben contestar si han vivenciado alguna de las conductas que refiere el ítem entre tres opciones de frecuencia en la semana escolar (ej.: "durante esta semana otro chico/a amenazó con pegarme": nunca/una vez/ más de una vez). La consistencia interna y fiabilidad del cuestionario se produjo mediante el alfa de Cronbach obteniendo .867 de 38 elementos analizados. 
Para el cálculo del índice de victimización y el índice de agresión general se siguió el procedimiento referenciado por Cerezo Ramírez (1998) para el instrumento mencionado que, de los ítems que contiene el cuestionario, extrae seis indicadores directamente relacionados con el Bullying para la construcción de los índices. De acuerdo al cuestionario utilizado, los ítems que se utilizaron para la extracción de los índices son los siguientes: "me dio patadas o golpes de puño"; "amenazó con pegarme"; "trató de quitarme plata"; "trató de lastimarme o herir mis sentimientos"; "me rompió algo"; y "trató de pegarme". La obtención del índice de victimización se logra mediante un cálculo que contabiliza las veces que cada uno de los 6 ítems es respondido con la categoría "más de una vez", extrayendo el porcentaje para cada ítem y hallando la media aritmética de los seis. Para el caso de esta investigación, se calculó el porcentaje correspondiente a la cantidad de respuestas en la categoría "más de una vez" (total de ítems con respuesta de 3 dividido 6 y multiplicado por 100), para que luego al momento de realizarse los cálculos estadísticos se calculara la media aritmética del porcentaje de acuerdo a la cantidad de niños en cada grupo analizado. Para el cálculo del indice de agresión general se utiliza un esquema parecido, se extraen los porcentajes de respuestas de los niños en la categoría "más de una vez" para cada uno de los 6 ítems; luego se procede igual pero con respecto a la categoría "una vez" para cada uno de los 6 ítems; posteriormente se juntan los 12 porcentajes y se dividen por 12. En este caso, se calculó la frecuencia de respuestas en la categoría "más de una vez" y "una vez" (total de ítems con respuesta 2 o 3 ) y se lo dividió por 12. La proporción se multiplicó por 100 para obtener el porcentaje. Luego en los cálculos estadísticos se considera el n por grupo para obtener el valor promedio en cada uno de ellos.

Para el tercer estudio de campo, se utilizó una muy breve encuesta en la que se preguntó a los niños sobre los siguientes temas: motivos que desencadenan las agresiones en la escuela, la presencia o no de adultos cuando se generan, los lugares en que generalmente se producen, entre otras opiniones.

\section{Procedimiento}

El cuestionario "Mi vida en la escuela" se aplicó en instituciones de las localidades antes mencionadas durante los horarios de clases y con la autorización de la Dirección de las escuelas, su llenado fue totalmente anónimo y voluntario por parte de los niños. La toma fue realizada por miembros del equipo de investigación familiarizados con el instrumento. Su aplicación se efectuó a mediados del segundo cuatrimestre de clases del año y los días viernes, de manera que los niños ya hubieran transitado la semana escolar. El tiempo que los niños tardaron en responder el cuestionario varió en función de los grados y escuelas, aunque en general osciló 
entre los 20 y 30 minutos (en los grados 7 mo. 8vo. y 9no.) y entre los 30 y 45 minutos (en los grados 3ro. 4to. y 5to.). En todos los trabajos se cumplimentó con el procedimiento del consentimiento informado de los padres o tutores del niño con la correcta expresión de los fines, objetivos de la investigación, el uso de los datos, los responsables del trabajo, instituciones participantes, como también la voluntariedad de participar, la reserva de confidencialidad y anonimato.

El tratamiento de la información cuantitativa producida por el cuestionario aplicado se analizó con software específico para el tratamiento de datos (IBM-SPSS, Statistical Package for the Social Sciences). La información más cualitativa producida por la encuesta a niños se elaboró con diversas técnicas de procesamiento, mediante la utilización del Software de análisis cualitativo de datos Nvivo (QSR Internacional).

\section{Resultados}

Se analizó la muestra tomando las localidades para evaluar si existían diferencias estadísticamente significativas entre las mismas y para realizar esta comprobación se realizaron dos pruebas ANOVA one-way con post-hoc Tukey-b. Tal prueba mostró resultados estadísticamente significativos en ambos índices: índice de victimización, $F(2,860)=11.81, p<.001$; índice de agresión general, $F(2,794)=11.26, p<.001$. La prueba post-hoc indicó que en ambos casos en Santa Cruz hay niveles de bullying significativamente mayores que en los otros dos lugares (ver Tabla 1).

Tabla 1.

Índices de Victimización y Agresión General en escuelas de localidades de la Zona Centro de la Pcia. de Santa Cruz.

\begin{tabular}{lccc}
\hline & $\begin{array}{c}\text { San Julián } \\
(n=350)\end{array}$ & $\begin{array}{c}\text { Piedrabuena } \\
(n=229)\end{array}$ & $\begin{array}{c}\text { Santa Cruz } \\
(n=289)\end{array}$ \\
\hline Índice de Victimización & $9.74_{\mathrm{A}}$ & $11.57_{\mathrm{A}}$ & $17.69_{\mathrm{B}}$ \\
Índice de Agresión General & $10.95_{\mathrm{A}}$ & $13.08_{\mathrm{A}}$ & $16.55_{\mathrm{B}}$ \\
\hline
\end{tabular}

Nota: Letras iguales significan conjuntos homogéneos. Datos obtenidos durante los años 2005/2006 de una muestra no probabilística de 868 niños

Para verificar si existía una diferencia en los índices de victimización y agresión de acuerdo con la edad se calcularon dos pruebas ANOVA one-way con post-hoc Tukey-b. En el caso del índice de agresión el resultado fue estadísticamente significativo, $F(6,701)=2.32, p=.031$. El análisis post-hoc indicó que la 
diferencia se encontraba entre el grupo de 10 años en donde se visualizó un mayor índice de agresión en comparación con el grupo de 14 años en donde el índice fue menor. En el resto de los grupos el índice tuvo un valor intermedio (ver Tabla 2). En el caso del índice de victimización el resultado estuvo al límite de la significancia estadística, $F(6,762)=2.07, p=.055$, y en análisis post-hoc indicó una diferencia estadísticamente significativa nuevamente entre el grupo de 10 años y el de 14 años que también daba cuenta de un pico de victimización a los 10 años y una baja significativa en el grupo de 14 años. El resto de los grupos etarios estaban en un intermedio de victimización entre ellos.

Tabla 2.

Índices de Victimización y Agresión General en escuelas de localidades de la Zona Centro de la Pcia. de Santa Cruz distribuido por edad

\begin{tabular}{cccccccc}
\hline & $\begin{array}{c}8 \text { años } \\
(n=108)\end{array}$ & $\begin{array}{c}9 \text { años } \\
(n=125)\end{array}$ & $\begin{array}{c}10 \text { años } \\
(n=103)\end{array}$ & $\begin{array}{c}11 \text { años } \\
(n=110)\end{array}$ & $\begin{array}{c}12 \text { años } \\
(n=108)\end{array}$ & $\begin{array}{c}13 \text { años } \\
(n=93)\end{array}$ & $\begin{array}{c}14 \text { años } \\
(n=126)\end{array}$ \\
\cline { 2 - 8 } $\begin{array}{c}\text { Índice de } \\
\text { victimización } \\
\begin{array}{c}\text { Indice de } \\
\text { agresión } \\
\text { Gral. }\end{array}\end{array}$ & $10.37_{\mathrm{AB}}$ & $12.91_{\mathrm{AB}}$ & $17.53_{\mathrm{B}}$ & $11.36_{\mathrm{AB}}$ & $13.53_{\mathrm{AB}}$ & $11.61_{\mathrm{AB}}$ & $9.28_{\mathrm{A}}$ \\
\hline
\end{tabular}

Nota: Letras iguales significan subconjuntos homogéneos

Para visualizar si existían diferencias de sexo en los índices se calculó la prueba $t$ de Student para cada índice. De este modo, resultó para el índice de victimización, $t(855)=4.67, p<.001$; para el índice de agresión general, $t(790)=3.10, p=.002$., lo que expresa diferencias estadísticamente significativas para ambos. Se puede verificar que los índices tanto de agresión general como de victimización son bastante menores en el caso de las niñas que si nos referimos a los varones. Las niñas reciben menos agresiones, pero debido a las características del instrumento utilizado no fue posible saber si las reciben de las niñas o de los niños.

\section{Tabla 3.}

Índices de Victimización y Agresión General según diferencias por sexo

\begin{tabular}{lcc}
\hline & $\begin{array}{c}\text { Varones } \\
(n=417)\end{array}$ & $\begin{array}{c}\text { Mujeres } \\
(n=445)\end{array}$ \\
\hline Índice de Victimización & 15.40 & 9.21 \\
\hline Índice de Agresión Gral. & 14.52 & 11.57 \\
\hline
\end{tabular}

Nota: Datos obtenidos de una muestra no probabilística de 868 niños 
El estudio de campo realizado en el 2009 en la localidad de San Julián aplicó las mismas técnicas y el mismo cuestionario, aunque sólo en el tercer ciclo de la EGB. A fin de establecer si existían diferencias significativas en los índices por grado escolar (7mo., 8vo. y 9no.) se compararon los resultados de las submuestras. Para esta comprobación se realizaron dos pruebas ANOVA one-way con post-hoc Tuckey-b. Tal prueba no mostró resultados estadísticamente significativos en ninguno de los dos índices (ver tabla 4).

\section{Tabla 4.}

Índices de Victimización y Agresión General en una escuela de la Zona Centro de la Pcia. de Santa Cruz distribuido por grados del 3er. ciclo

\begin{tabular}{lccc}
\hline & $\begin{array}{c}7 \text { mo.Grado } \\
(n=108)\end{array}$ & $\begin{array}{c}8 v o . \text { Grado } \\
(n=94)\end{array}$ & $\begin{array}{c}\text { 9no. Grado } \\
(n=62)\end{array}$ \\
\hline Indice de victimización & $13.58_{\mathrm{A}}$ & $8.79_{\mathrm{A}}$ & $9.14_{\mathrm{A}}$ \\
\hline Indice de agresión general & $14.02_{\mathrm{A}}$ & $10.98_{\mathrm{A}}$ & $10.98_{\mathrm{A}}$ \\
\hline
\end{tabular}

Nota: Letras iguales significan conjuntos homogéneos. Datos obtenidos de una muestra no probabilística de 264 niños durante el año 2009

Para el caso del análisis de las diferencias por sexo respecto de los índices resultantes de la muestra tomada en los estudios del 2009 se calculó la prueba $t$ de student para cada índice resultando para el índice de victimización, $t(262)=3.00$, $p=.001$; para el índice de agresión general, $t(252)=3.12, p=.030$. Los resultados al igual que en el estudio del 2006 mostraron diferencias estadísticamente significativas en los índices de las mujeres y los varones, confirmando nuevamente que son estos últimos los que sufren mayormente prácticas de bullying.

Tabla 5.

Índices de Victimización y Agresión General según diferencias por sexo

\begin{tabular}{lcc}
\hline & $\begin{array}{c}\text { Varones } \\
(n=139)\end{array}$ & $\begin{array}{c}\text { Mujeres } \\
(n=125)\end{array}$ \\
\hline Índice de Victimización & 13.98 & 7.33 \\
\hline Índice de Agresión Gral. & 14.40 & 9.68 \\
\hline
\end{tabular}

Nota: Datos obtenidos de una muestra no probabilística de 264 niños

Se analizaron los comportamientos más comunes vinculados a las prácticas de intimidación. Los datos surgidos en las distintas localidades tienen como denominador común que un importante número de los niños manifestaron que 
durante la semana les han intentado pegar más de una vez. Lo mismo se puede decir de las amenazas que recibieron y también respecto de haber recibido golpes de puño o patadas. El ser golpeados más de una vez en la semana ocupa en los ítems mencionados siempre un lugar preponderante en todas las escuelas. Para evaluar la existencia de asociación del sexo con los tipos de agresión se calcularon pruebas chi-cuadrado, surgiendo diferencias estadísticamente significativas en los ítems: Recibió patadas o golpes, $\chi^{2}=17.867, p<001$, Amenazaron con pegarle, $\chi^{2}$ $=12.169, \mathrm{p}<001, \mathrm{y}$ Trataron de pegarle, $\chi^{2}=12.628, \mathrm{p}<001$. Los demás ítems no alcanzan el límite de significancia estadística dep $<.05$. De lo expuesto se desprende que existe una asociación entre el sexo y comportamientos como recibir golpes, amenazas y ser objeto de intentos de agresión física directa. No surgió una relación significativa respecto del sexo en prácticas como herir los sentimientos, quitarles plata y romper útiles escolares u otros objetos personales (ver tabla 6).

\section{Tabla 6.}

Niños y niñas que recibieron agresiones varias veces durante la semana en las escuelas

\begin{tabular}{lcc}
\hline Tipo de agresión & $\begin{array}{c}\text { Varones } \\
(n=445)\end{array}$ & $\begin{array}{c}\text { Mujeres } \\
(n=417)\end{array}$ \\
\hline Recibió patadas o golpes & 86 & 38 \\
\hline Amenazaron con pegarle & 76 & 38 \\
\hline Trataron de quitarle plata & 15 & 7 \\
\hline Hirieron sus sentimientos & 63 & 46 \\
\hline Le rompieron algo & 63 & 43 \\
\hline Trataron de pegarle & 99 & 54 \\
\hline
\end{tabular}

Nota: Se tomaron sólo los indicadores de acoso que se utilizan para la construcción de los índices de victimización. Datos obtenidos de una muestra no probabilística de 868 niños

Durante el año 2010, como se mencionó en el apartado de los participantes, se realizó una muy breve encuesta en la que se preguntó a los niños/as sobre cuáles son los motivos por los que se desencadenan las agresiones en la escuela. Esta vez se consultó a $n=564$ niños asistentes a las escuelas de EGB y Polimodal de poblaciones de la región central de la Pcia. de Santa Cruz, en este caso fueron las localidades Pto. Santa Cruz, Piedrabuena y Gobernador Gregores.

En primer lugar, según datos obtenidos a nivel general, las situaciones que más comúnmente originan las agresiones tienen que ver con los insultos a un compañero o compañera. Los niños manifiestan que ser insultados es uno de los principales motivos desencadenantes de agresiones físicas. En segundo lugar, el otro motivo 
que aparece es el de "pegarse", un gran número de niños reacciona agresivamente por haber recibido golpes; ésta conducta suele ser en respuesta de defensa, un comportamiento reactivo surgido de la agresión previa o la provocación. En el segundo ciclo de la EGB la razón más importante de las agresiones son los insultos, y pasan a un segundo plano las cuestiones que tienen que ver con respuestas a las agresiones físicas como empujarse, etc. En el tercer ciclo crece en importancia el motivo relacionado con las peleas o agresiones "por un chico" o "una chica". Aunque los insultos siguen manteniendo mayor importancia.

Otro de los aspectos a destacar es que gran parte de las situaciones se produce durante la clase (dicho por los propios niños), bajo la presencia del profesor o en su ausencia en el aula. Surge con bastante importancia el uso de las herramientas de Internet para las prácticas de provocación, exposición del otro, etc. Esto demuestra que la emergencia y uso de este tipo de dispositivos para el acoso y la persecución está resultando cada vez más frecuente.

Se analizó la posibilidad de asociación del sexo con las conductas que originan más agresiones entre los escolares. Para ello se utilizó el cálculo de chi-cuadrado, surgiendo diferencias estadísticamente significativas en los ítems: ser insultado, $\chi^{2}=$ 8.916, $\mathrm{p}=003$, recibir golpes $\chi^{2}=10.907, p=001$, ser molestado, $\chi^{2}=4.254, p=039$, ser discriminado, $\chi^{2}=4.154, p=.042$, disputar una chica o un chico, $\chi^{2}=4.355, p=$ 037. El indicador recibir empujones y trabadillas no alcanzó el límite de significancia estadística $p<.05$. Queda reflejado que para los varones los insultos, los golpes y ser molestado resultan ser las prácticas más frecuentes que originan más agresiones. En cambio, en el caso de las mujeres es más típico que las agresiones se produzcan por la discriminación o debido a la disputa por una chica o un chico (ver tabla 7).

Tabla 7.

Conductas que comúnmente originan más agresiones entre los escolares

\begin{tabular}{lcc}
\hline Ser insultado & $\begin{array}{c}\text { Varones } \\
(n=264)\end{array}$ & $\begin{array}{c}\text { Mujeres } \\
(n=300)\end{array}$ \\
\hline Recibir golpes & 145 & 127 \\
\hline Ser molestado & 87 & 62 \\
\hline Recibir empujones y trabadillas & 123 & 114 \\
\hline Ser discriminado & 56 & 52 \\
\hline Disputar una chica o chico & 32 & 55 \\
\hline
\end{tabular}

Nota: Datos obtenidos sobre una muestra no probabilística de 564 niños durante el año 2010 


\section{Comentarios}

A modo de comentario general podría decirse que las conductas agresivas se distinguen en cada uno los ciclos de la Educación, tienen algunos denominadores comunes pero en parte difieren y tienen características propias en cada localidad, variando según el escenario particular de cada escuela.

No resulta posible relacionar los índices obtenidos aquí con otros contextos, ya que no se encontraron trabajos comparables en Latinoamérica que hayan usado tales índices en la misma época. Compararlos con los que se han obtenido en países de Europa donde se han realizado estudios similares no parece ser una buena opción debido a las características contextuales muy diferentes a las de nuestro país (caso de Noruega, Finlandia, Suecia).

El valor de tales índices radica no sólo en lo que expresa a nivel de la región, sino también, que pueden ser utilizados en estudios futuros para poder apreciar la evolución y realizar un seguimiento adecuado del tema de la Intimidación en las escuelas.

Respecto a las diferencias de sexo que se expresan en las diversas prácticas de niños y niñas, surge que amenazar con pegar, dar patadas o golpes de puño, tratar de pegar, y otras conductas de agresión directa son más típicas de los varones que de las mujeres. La agresión directa parece ser una práctica más común en los niños que en las niñas, con excepción de los empujones o trabadillas que parecen ser comportamientos más naturalizados de la población escolar en general.

Una de las explicaciones que se escucha con frecuencia entre los docentes vincula el incremento de la violencia y la agresión con los repitentes, se supone que involucra también la problemática de la sobreedad, es decir, la convivencia de niños mayores con otros mucho más pequeños que presentan otras motivaciones, intereses, etc. Puede tener sustento esta explicación en algunos cursos particulares, pero no es correcto generalizar, en las muestras estudiadas no se han encontrado datos concretos que confirmen la hipótesis de que en tales cursos las prácticas de intimidación sean mucho mayores. Hay cursos en los que el número de repitentes es muy importante pero los índices no son altos.

Con respecto a los motivos que originan las agresiones, se puede decir que algunos se mantienen a lo largo de los diversos años de la EGB, como lo es el caso de insultar y pegar a un compañero. Otro ejemplo muy concreto es el del empujón y trabadilla. Los datos obtenidos mediante el cuestionario de intimidación en las escuelas, informan que una de las prácticas que aparece muy naturalizada es la de la "zancadilla o trabadilla", que generalmente viene acompañada de un empujón previo para que el niño se caiga. Podría decirse que la práctica de la trabadilla es una acción que, si bien pretende efectuarse por diversión entre los niños, no deja de ser una actitud de agresión que termina siendo una de las mayores razones por 
las cuales se originan peleas, según la opinión de los propios escolares.

Resulta relevante trabajar con los docentes y personal de apoyo a la docencia para que se tengan muy en cuenta estas actitudes de los niños, ya que al ocurrir con tanta frecuencia, como surge de los datos, no es posible que no sean vistos por los docentes y demás personal. Más bien hay una cierta naturalización de tal comportamiento que parece no penarse de manera seria o no percibirse como una conducta susceptible de marcar con una llamada de atención, lo que puede derivar en el peligro de la invisibilización de ciertas acciones violentas.

Respecto de los resultados de los estudios presentados en este escrito, se puede decir que varios de los datos regionales coinciden con lo que muchos de los trabajos citados en la introducción plantean acerca del fenómeno del acoso escolar. Por ejemplo si nos referimos a las prácticas de hostigamiento más comunes entre los escolares, se pueden confirmar las diferencias entre las de los niños y las de las niñas; coincidiendo con otros estudios, los varones optan por la violencia de características físicas y directas, mientras que las mujeres presentan comportamientos más vinculados con las conductas indirectas de exclusión y de aislamiento social de las víctimas (Hopkins et al. 2013; Pörhölä \& Kinney, 2010; Voors, 2006).

Las prácticas de intimidación están siendo acompañadas con el surgimiento de estrategias relativamente recientes que atraviesan las fronteras escolares, entre las que se destacan: el uso de las herramientas que proporciona la WEB, como es el caso del Chat, los blogs, la publicación de fotografías o videos (que comprometen o exponen a algún compañero), y el uso de graffitis en las inmediaciones de la escuela. Espacios que pueden usarse para generar discordia entre grupos, para hablar mal del otro, generar rumores injuriosos, provocar exclusión social o grupal de alguien, etc. Estas nuevas modalidades, además de ejercer provocación que podría culminar en violencia (en un espacio en el que los niños se encuentren), también pueden, dependiendo del caso, convertirse en formas de intimidación o acoso.

Hay conductas de acoso entre pares que se originan en ámbitos distintos de los escolares, pero muchas veces se trasladan y continúan dentro del contexto escolar. Los lugares de origen pueden ser los clubes, los barrios, gimnasios, boliches bailables y pubs, entre otros ámbitos. Es menester hacer un intento por comprender la fuente de tales conflictos, que si bien son de diversa índole, en algunas situaciones se reiteran los protagonistas.

Un fenómeno que se ha podido apreciar es el que hemos llamado "Efecto de Acorralamiento", que presenta impacto a nivel de la salud mental de los niños. Este es producido por una característica que poseen en común las localidades pequeñas del interior del país: la falta de anonimato. A diferencia de las grandes zonas urbanizadas, los ciudadanos de las pequeñas poblaciones tienen un conocimiento muy preciso de sus vecinos y de los habitantes del lugar. Las zonas de concurrencia, de recreación, práctica de deportes, actividades culturales, 
etc. son lugares de encuentro de la mayoría de los vecinos de la localidad. Esta situación se traduce en una ventaja para los victimarios y en una extrema vulnerabilidad para sus blancos o víctimas. En términos concretos un niño Bull puede conocer todos los posibles movimientos de su víctima en el entorno en que habita, sabe los lugares a los que concurre fuera de la escuela, los deportes que practica, etc., incluso los horarios en que lo hace. El niño acosado también intuye que su acosador sabe de ello, y como efecto de esta circunstancia la vivencia del hostigamiento se prolonga más allá de las fronteras escolares, aun cuando ello pueda no ocurrir en la realidad. La sensación de persecución se convierte entonces en una circunstancia vital con la consecuencia de que los niveles de atemorización se incrementan notablemente provocando un fuerte impacto en la salud mental del niño. A este fenómeno lo hemos caracterizado como el "efecto acorralamiento", que refiere al significado de encerrar el ganado en el corral: por las características que se describieron el niño perseguido se percibe encerrado o rodeado con la sensación de no poder escapar por ningún flanco de la intimidación; tal circunstancia deja al sujeto confundido y sin poder reaccionar.

Las futuras líneas de indagación e investigación se orientarán a estudiar diversas dimensiones de nuevas prácticas de violencia, éstas significan un importante desafío por el impacto que pueden presentar en el sujeto y comprometen a pensar el tema desde diversos campos, psicológico, educativo, jurídico y sociológico. No se pueden desconocer los aspectos positivos que la tecnología ha permitido en los últimos años, el uso que los niños y adolescentes hacen de ella y el impacto que tiene en la construcción de su identidad como menciona Morduchowics (2012). Pero junto a ello, es posible verificar el surgimiento de nuevas conductas, algunas caracterizadas como "Cyberbullying", y que tienen como contexto lo que Pizzo (2009) describe como "nuevos escenarios que se ofrecen a la infancia y la adolescencia". El incremento del uso de los recursos tecnológicos por parte de la población infantil y adolescente en la última década, el acceso cada vez mayor, la facilidad con que aprenden a usarlos y la comodidad con que se manejan con ellos, trajo al mismo tiempo una acentuación en las prácticas violentas en ese contexto virtual. El acoso o la intimidación están presentes en estos nuevos escenarios. Investigadores como Kowalski, Limber y Agatston (2010) o Mishna, Khoury-Kassabri, Gadalla y Daciuk (2012), están produciendo trabajos muy interesantes para comprender las características de este fenómeno caracterizado como Cyberbullying. Se entienden como "nuevas formas de violencia" las prácticas que se producen mediante recursos como la mensajería instantánea, el correo electrónico, los chat, las blogs, fotologs, redes sociales, todos los relacionados con tecnologías de comunicación digital, por medio de dispositivos como los ordenadores de escritorio y portátiles, tablets, smartphones, 
y otros, que posibilitan tales comunicaciones. Los niños y adolescentes hacen múltiples usos de las herramientas disponibles en la Web; muchos son realmente positivos, pero interesa aquí particularmente estudiar la práctica específica con fines de hostigamiento (Kowalsky \& Limber, 2013) y los efectos psicológicos que provoca en quienes la sufren (Cankaya \& Tan, 2011).

La investigación de este problema data de la última década, y en los últimos años se fueron incrementando los trabajos sobre el tema, seguramente debido al aumento real de las víctimas de estas violencias. Existen numerosos "agujeros negros" en el conocimiento de la temática, por causa de variadas circunstancias, por ejemplo: una tiene que ver con que su emergencia es bastante reciente; y otra con la permanente innovación de las herramientas y dispositivos, que permiten prácticas que hace algunos años no eran imaginables. Los estudios existentes sobre el tema mayoritariamente señalan que la cantidad de niños y adolescentes víctimas de algún tipo de violencia por estos medios ha crecido en los últimos años (Castro Santander, 2012). Los psicólogos en la práctica clínica frecuentemente se topan con casos de niños, adolescentes y adultos que sufren por consecuencia de haber sido objeto de estas nuevas formas de violencia.

Otra de las consecuencias de hostigamiento es la sensación de culpa y pesar, que suele ser un efecto bastante común que se presenta en los "espectadores" (Harris \& Petrie, 2006). Los espectadores son quizá los actores menos estudiados del problema, siendo que en muchas ocasiones favorecen la reproducción de la violencia. Los estudios sobre los espectadores de la violencia deberían adquirir mayor protagonismo en los próximos tiempos.

Hay dos aspectos más que pueden ser futuras líneas de indagación, en primer lugar, recientes trabajos encuentran diferencias importantes entre los tipos de prácticas de violencia de las mujeres y de los varones, y también diferencias en los efectos entre el bullying tradicional y el cyberbullying (Kowalsky \& Limber, 2013; Kowalsky et al. 2010). En segundo lugar, en investigaciones regionales en localidades de baja población urbana se ha encontrado que el anonimato suele ser menos frecuente, un porcentaje importante de niños que sufrieron este tipo de violencia han manifestado conocer la identidad del agresor (Pautasso, 2006; Pautasso, Carnota, \& García, 2013). Es posible suponer que los efectos psicológicos en unos y otros casos también pueden ser diversos.

Para culminar, en el escenario planteado sobre el tema de la violencia e intimidación entre los niños, el desafío más significativo tiene que ver con cómo construir Dispositivos y mecanismos que permitan alertar tempranamente de las poblaciones más vulnerables y atender con la mayor urgencia posible tales situaciones.

La construcción de un "Dispositivo de Alerta Temprana"-DAT, se compondría de una estructura de sensores para la detección de los primeros emergentes de una 
problemática determinada y de la evolución de la misma en el escenario en que se presenta. Constituye un mecanismo esencial dentro del sistema que se podría proponer y el primer eslabón del mismo. Se lo puede imaginar como un conjunto de terminales "sensitivas" que mostrarían los indicadores en determinadas áreas sensibles o vulnerables y que proporcionarían información del estado actual, su evolución histórica y los factores más relevantes de la problemática particular del Bullying. Una observación continuada de esos indicadores permitiría percibir qué tendencias de esos factores pueden ser tomadas como oportunidades o como amenazas. El dispositivo de alarma temprana es la herramienta para tomar decisiones basadas en una interpretación más objetiva del entorno, en un pronóstico, en las tendencias detectadas y en su posible evolución futura. La puesta en marcha de estos dispositivos en un programa organizado permitiría ir adaptando de modo continuado las políticas educativas que pudieran plantearse alrededor de la temática de la intimidación y aportaría a la prevención. La información suministrada por estos dispositivos contribuye al conocimiento sostenido de la problemática y a la planificación de las acciones de una manera más eficiente y coordinada, evitando el abordaje improvisado cuando aparece un emergente particular. Los dispositivos apoyados en técnicas de alarma temprana pueden ser considerados como una herramienta de "pronóstico" elaborada dentro de una estrategia prospectiva.

Por DAT - Dispositivo de Alerta Temprana se puede entender un sistema mediante el cual las organizaciones institucionales de la comunidad obtendrán información valiosa sobre determinadas problemáticas relacionadas con la infancia. La función del DAT consiste en:

- identificar una problemática en el momento oportuno, para poner en funcionamiento el sistema de prevención, atención, etc.

- procesar las informaciones pertinentes de modo que pueda formularse un planteamiento concreto de la situación problema;

- construir un cierto diagnóstico a partir de la información, que provea una manera básica de ayuda tanto en la decisión de medidas a tomar como en acciones que permitan aprovechar las oportunidades abiertas o escapar de los potenciales riesgos de la situación problema;

- poner en funcionamiento la estructura operativa del Dispositivo de Intervención Temprana - DIT.

Obtener una adecuada información sobre la situación del problema de intimidación en las escuelas no brinda utilidad si no se dispone de mecanismos eficientes de intervención y atención de tales situaciones. Por ello, se considera clave el diseño y elaboración de dispositivos que apunten a estrategias de rápida intervención. En tales dispositivos siempre se debe pensar en el trabajo 
colaborativo entre instituciones, acciones en redes coordinadas que respeten la órbita de competencia de cada institución, la responsabilidad que les compete y la capacidad operativa de cada una, así como sus limitaciones y potencialidades.

Es imprescindible la participación de todos los actores y sectores involucrados en el escenario escolar; alumnos, docentes, directivos y padres.

Las dificultades para abordar e intervenir con rapidez y eficacia en la resolución de problemas y casos individuales y grupales debería superarse constituyendo dispositivos que preserven a los niños involucrados, garantizando la atención de su salud, operando para su recuperación y planificando para prevenir situaciones futuras.

Como desafío, sería relevante la implementación de un programa que apunte a la organización e implementación de Dispositivos de Intervención TempranaDIT. Tal programa debería orientarse a la organización de una estructura operativa de intervención, constituyendo un gabinete profesional interdisciplinario para abordar y atender las situaciones emergentes. Asimismo, debería articularse con espacios en Red más amplios, compuestos por otras instituciones operantes en los problemas de la infancia, ya que muchos de los problemas emergentes trascienden las fronteras escolares y atraviesan las cuestiones familiares y comunitarias.

\section{Referencias}

Bauman, S., Toomey R. \& Walker J. L. (2013). Associations among bullying, cyberbullying, and suicide in high school students. Journal of Adolescence, 36(2), 341350. doi:10.1016/j. adolescence.2012.12.001

Bejarano, A. \& Recio, E. (2000). Centro de Documentación e Información Educativa sobre Violencia Escolar. Diseminación selectiva de la información $N^{\circ}$ 7. Resistencia.

Cankaya, İ. H. \& Tan, C. (2011). Effect of cyber bullying on the distrust levels of preservice teachers: considering internet addiction as a mediating Variable. Procedia Computer Science, 3(C), 1353-1360. doi:10.1016/j.procs.2011.01.015

Castro Santander, A. (2012). Conflictos en la era de la escuela digital: Tecnología $y$ violencia. Buenos Aires: Bonum.

Catala, S. (2008). Violencia en las escuelas. Un relevamiento desde la mirada de los alumnos. Observatorio Argentino de violencia en las escuelas. Buenos Aires: Ministerio de Educación. 
Cerezo Ramírez, F. (1998). Conductas agresivas en la edad escolar. Madrid: Pirámide.

Cerezo Ramírez, F. (2001). La violencia en las aulas. Análisis y propuestas de intervención. Madrid: Pirámide.

Garay, L. \& Gezmet, S. (2001). Violencia en las escuelas. Fracaso educativo. Publicación del Programa de Análisis Institucional de la Educación. Centro de Investigaciones de la Facultad de Filosofía y Humanidades. Córdoba: UNC.

Giberti, E. (13 de noviembre de 2000). Formas de violencia escolar. Clarín. Recuperado de http://edant.clarin.com/diario/2000/11/13/o-02501.htm

Harris, S. \& Petrie, G. (2006). El acoso en la escuela. Los agresores, las víctimas y los espectadores. Barcelona: Paidós Educador.

Hopkins, L. Taylor, L. Bowen, E. \& Wood, C. (2013). A qualitative study investigating adolescents' understanding of aggression, bullying and violence. Children and Youth Services Review, 35(4), 685-693. doi:10.1016/j. childyouth.2013.01.012

Kowalski, R. M. \& Limber, S. P. (2013). Psychological, Physical, and Academic Correlates of Cyberbullying and Traditional Bullying. Journal of Adolescent Health, 53(1), S13-S20. doi:10.1016/j.jadohealth.2012.09.018

Kowalski, R. M., Limber, S. P,, \& Agatston, P. (2010). Cyber Bullying: El acoso escolar en la era digital. Desclee de Brouwer: Colección Amae.

Lejarraga, A. Pampurro, L. Zajac, J. \& D'odorico, C. (2011). Cómo convivir en la escuela. Una crítica al concepto de bullying en las escuelas. Documento de Trabajo. Observatorio Argentino de Violencia en las Escuelas. Buenos Aires: Ministerio de Educación.

Miguez, D. (2008). Violencias y conflictos en las escuelas. Buenos Aires: Paidós.

Mishna, F. Khoury-Kassabri, M. Gadalla, T. \& Daciuk, J. (2012). Risk factors for involvement in cyber bullying: Victims, bullies and bully-victims. Children and Youth Services Review, 34(1), 63-70. doi:10.1016/j.childyouth.2011.08.032

Morduchowics, R. (2012). Los adolescentes y las redes sociales: La construcción de la identidad juvenil en internet. Buenos Aires: Fondo de Cultura Económica. 
Noel, G. (2009). Violencia en las escuelas desde una perspectiva cualitativa. Observatorio Argentino de violencia en las escuelas. Buenos Aires: Ministerio de Educación de la Nación.

Olweus, D. (1998). Conductas de acoso y amenaza entre escolares. Madrid: Morata.

Pautasso, N. (2006). La intimidación entre los niños en edad escolar. Estudio sobre las sutiles prácticas de violencia en el ámbito de la EGB. En memorias del Segundo Encuentro de investigadores en psicología del Mercosur. Paradigmas, métodos y técnicas. Ediciones de la Facultad de Psicología: Universidad de Buenos Aires. ISSN 1667-6750.

Pautasso, N. Carnota L. \& García, S. (2013). Nuevas formas de violencia e intimidación entre los niños en edad escolar. Una descripción de algunas prácticas violentas. En A. Tromboli (Ed.), Salud Mental: Interdisciplina e Inclusión Social Como Ejes De Intervención. AASM.

Pizzo, M. E. (2009). El desarrollo de los niños en edad escolar. Ficha de estudio de la Facultad de Psicología. UBA. Inédito.

Pörhölä, M. \& Kinney, T. (2010). El Acoso. Contexto, consecuencias y control. España: Aresta.

Sabucedo, J. \& Sanmartin, J. (2007). Los escenarios de la violencia. Barcelona: Ariel.

Sharp, S. \& Smith, P.K. (1994). Tackling bullying in your school: A practical handbook for teachers. Londres: Routledge.

Voors, W. (2006). Bullying. El acoso escolar. Buenos Aires: Oniro. 
